\section{Präventive Monotherapie mit Montelukast versus DNCG bei Kindern mit leichtem Asthma bronchiale}

\author{
Ergebnisse einer explorativen Pilotstudie
}

Zusammenfassung: Hintergrund: Bisher wird Montelukast im Kindesalter als „add-on“-Präparat in Ergänzung zu inhalativen Steroiden eingesetzt. Es sollte überprüft werden, ob Montelukast als Monotherapie zur Dauerprophylaxe bei Kindern mit leichtem Asthma bronchiale geeignet und einer regelmäßigen Inhalationstherapie mit DNCG überlegen ist. Methode: 20-wöchige offene, randomisierte Cross-over-Studie an 20 Kindern im Alter zwischen 6 und 14 Jahren mit Asthma bronchiale und Bedarf einer prophylaktischen Medikation (über 6 Wochen mehr als zweimal wöchentlich Bedarf an Beta-Sympathomimetika). Nach 2-wöchiger Runin-Periode Therapiebeginn mit einmal $5 \mathrm{mg}$ Montelukast oder 3 Inhalationen à $20 \mathrm{mg}$ DNCG pro Tag. Nach 8 Therapiewochen erfolgte eine 14-tägige Washout-Periode, um dann mit dem jeweils anderen Therapieregime über weitere 8 Wochen fortzusetzen. Ergebnisse: Die Therapie mit DNCG führte im Vergleich zur Runin-Periode zu einer signifikanten Besserung der FEV 1 (100,6 vs. 96,5\% des altersentsprechenden Solls, $\mathrm{p}<0,01)$ und des $\mathrm{MEF}_{25}(70,6$ vs. $59,1 \%, \mathrm{p}<0,05)$ in der Ruhelungenfunktion und nach Kaltluftprovokation ( $\mathrm{FEV}_{1}$ 97,2 vs. $91,2 \%, p<0,05 ; M_{25} 62,9$ vs. $\left.54,4 \%, p<0,01\right)$. Nach Einnahme von Montelukast fand sich beim $\mathrm{MEF}_{25}$ in der Ruhelungenfunktion eine signifikante Besserung von 59,1 auf $67,8 \%$ $(p<0,05)$. Beide Medikamente führten zu einer signifikanten Reduktion $(p<0,05)$ sowohl der Asthmabeschwerden am Tage, gemessen an einem subjektiven Beschwerdescore, als auch der abendlichen Peak-flow-Variabilität. Bei keinem der registrierten Endpunkte ließen sich relevante Unterschiede zwischen beiden Prüfsubstanzen ermitteln. Auch der Verbrauch von Bedarfsmedikation blieb unverändert. Schlussfolgerung: Vorliegende Pilotstudie zeigt, dass die prophylaktische Therapie mit Montelukast bei einfacherem Applikationsmodus eine vergleichbar gute Kontrolle des Asthma bronchiale ermöglicht wie DNCG. Studien, welche die Wirksamkeit von Montelukast als Monotherapie anhand größerer Fallzahlen belegen, sind jedoch erforderlich, um die Rolle von Leukotrienrezeptorantagonisten in der Therapie des kindlichen Asthma bronchiale ggf. neu zu definieren.

Preventive Monotherapy with Montelukast Versus DNCG in Children with Mild Asthma - Results of an Explorative Pilot Study: Background: Montelukast is used as an add-on medication with inhaled steroids in the therapy of childhood asthma. The aim was to determine the clinical effect of montelukast as a prophylactic therapy in mild asthma in comparison

Pneumologie 2001; 55: $231-237$

(c) Georg Thieme Verlag Stuttgart · New York ISSN 0934-8387

\author{
C. Liebke, C. Sommerfeld, U. Wahn, B. Niggemann \\ Abteilung für Pädiatrische Pneumologie und Immunologie, \\ Universitätsklinikum Charité - Medizinische Fakultät \\ der Humboldt-Universität zu Berlin
}

with inhaled sodium cromoglycate. Method: 20 children aged 6-14 years were treated in a 20-week open-labelled randomized cross-over design, starting after a 2-week run-in period with either montelukast or cromolyn for 16 weeks with a 2-week wash-out period between treatments. Results: Children treated with cromoglycate showed a significant increase of FEV 1 (100.6 vs. $96.5 \%, \mathrm{p}<0.01)$ and $\mathrm{MEF}_{25}(70.6$ vs. $59.1 \%, \mathrm{p}<0.05)$ in base line lung function and after cold air challenge ( $\mathrm{FEV}_{1} 97.2$ vs. $91.2 \%, \mathrm{p}<0.05 ; \mathrm{MEF}_{25} 62.9$ vs. $\left.54.4 \%, \mathrm{p}<0.01\right)$. Treatment with montelukast effected a significant increase $(p<0.05)$ in $\mathrm{MEF}_{25}$ from 59.1 to $67.8 \%$ in base line lung function alone. Both medications resulted in significant decreases $(p<0.05)$ in daytime asthma symptoms and evening peak flow variability. Comparing the two treatment substances no statistically significant differences could be registered in any endpoints including beta-agonist use. Conclusions: Both cromolyn and montelukast showed effective control of mild asthma in children; however, montelukast is more convenient in its application. Further studies are needed to determine the role of leukotriene receptor antagonists in childhood asthma.

\section{Einleitung}

Asthma bronchiale stellt die häufigste chronische Erkrankung im Kindesalter in den westlichen Industrienationen dar. Weltweit steigt die Prävalenz von kindlichem Asthma und die daraus resultierenden Krankenhausaufenthalte. Hauptziel der Asthmatherapie [1] besteht darin, das Auftreten von Symptomen und Exazerbationen zu verhindern und eine angemessene körperliche Aktivität zu ermöglichen, außerdem eine gut verträgliche Medikation anzubieten und nicht zuletzt den Erwartungen der Patienten bzw. deren Eltern gerecht zu werden.

In der prophylaktischen Langzeittherapie des kindlichen Asthma bronchiale findet neben den topischen Steroiden Dinatriumcromoglycat (DNCG) verbreitet Anwendung. In einer Reihe von Studien wurde die Wirksamkeit von DNCG belegt [2,3]. Nachteile sind jedoch regelmäßige, mehrmals tägliche Inhalationen und der damit verbundene Einfluss auf die Compliance. Es ist belegt, dass die Compliance bei oraler Asthmamedikation deutlich besser ist, als bei inhalativen Therapien [4]. Auf der anderen Seite muss insbesondere bei inhalativen Steroiden potentiell mit Nebenwirkungen wie Wachstumsretardierung [5] u.a. gerechnet werden. Daher sind neuere Therapien, die effektiv und gut verträglich und 
darüber hinaus leicht applizierbar sind, besonders für das Kindesalter von großem Interesse.

Zysteinyl-Leukotriene $\left(\mathrm{LTC}_{4}, \mathrm{LTD}_{4}, \mathrm{LTE}_{4}\right)$ sind die wichtigsten Leukotriene in der Pathogenese des Asthma bronchiale. Sie vermitteln eine Zunahme von Mukosapermeabilität und Mukusproduktion, eine Reduktion mukoziliärer Clearance, Kontraktion glatter Muskelzellen und Migration von Eosinophilen in die Atemwege [6-8]. Montelukast wirkt als potenter $\mathrm{LTD}_{4}$-Rezeptorantagonist und stellt aufgrund seiner Zulassung ab dem 6 . Lebensjahr für die pädiatrische Pneumologie derzeit den interessantesten Wirkstoff dieser neuen pharmakologischen Substanzklasse dar.

Inzwischen belegt eine Reihe von Studien die Wirksamkeit der Leukotrienrezeptorantagonisten zur Reduktion bronchialer Hyperreagibilität nach körperlicher Belastung [9] und zur Kontrolle des Asthma bronchiale [10-12]. Darüber hinaus vermindert Montelukast Früh- und Spätreaktionen auf Allergenprovokation $[13,14]$.

Auch für das Kindesalter ( 6 bis 14 Jahre) konnte eine Verbesserung der morgendlichen $\mathrm{FEV}_{1}$ [15] sowie eine Reduktion bronchialer Hyperreagibilität demonstriert werden [16].

Gemäß bisheriger Zulassung durch das Bundesinstitut für Arzneimittel wird Montelukast in der Pädiatrie als „add-on“Präparat in Ergänzung zu inhalativen Steroiden eingesetzt. Ziel vorliegender, kontrollierter Pilotstudie war es zu überprüfen, ob Montelukast auch bei Kindern mit leichtem Asthma bronchiale als medikamentöse Dauerprophylaxe geeignet ist und einer regelmäßigen Inhalationstherapie mit DNCG überlegen ist.

\section{Methodik}

\section{Patienten}

Eingeschlossen wurden 20 ambulant behandelte Kinder und Jugendliche im Alter zwischen 6 und 14 Jahren mit leichtem Asthma bronchiale, bei denen die Indikation einer prophylaktischen Medikation bestand (über 6 Wochen mehr als zweimal wöchentlich Bedarf an Beta-Sympathomimetika). Die Teilnehmer mussten in der Lage sein, in der Lungenfunktion reproduzierbare Ergebnisse hervorzurufen, regelmäßige Peakflow-Messungen vorzunehmen und ein „Asthmatagebuch“ zu führen. Ausgeschlossen wurden Patienten mit akuter Infektion der Atemwege, anderen akuten oder chronischen Erkrankungen oder Hyposensibilisierungstherapie. Abbruchkriterien waren akute Exazerbation, Hospitalisierung, unzureichender Behandlungserfolg, fehlende Compliance und Auftreten von Nebenwirkungen.

\section{Studiendesign}

Es wurde eine 20-wöchige offene, randomisierte Cross-overStudie an 20 Kindern und Jugendlichen mit Asthma bronchiale durchgeführt. Nach einer 14-tägigen Runin-Periode erhielten die Kinder je nach Randomisierung entweder Montelukast in Form einer $5 \mathrm{mg}$ Kautablette (Singulair ${ }^{\circledR}$, Fa. MSD Sharp \& Dohme, Haar) oder 3 Inhalationen mit je 20 mg DNCG über einen Kompressionsvernebler (Fa. Pari, Starnberg) pro Tag. Nach 8 Therapiewochen erfolgte eine 14-tägige Washout-
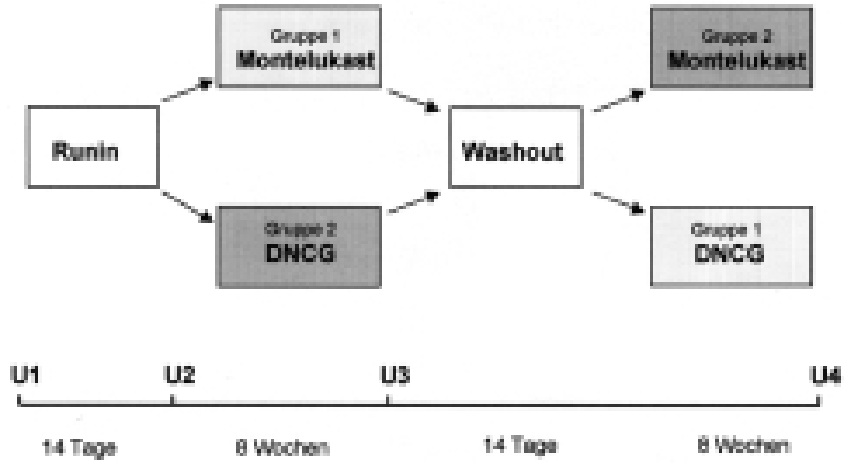

Abb. 1 Studiendesign. Untersuchungstermine sind als $U$ gekennzeichnet.

Periode, um dann mit dem jeweils anderen Therapieregime über weitere 8 Wochen fortzusetzen. Abb. 1 zeigt das Studiendesign mit den vier Vorstellungsterminen U1 bis U4. Nach einer ausführlichen Eingangsuntersuchung und Lungenfunktionsprüfung inklusive Bronchospasmolyse als U1 erfolgten während der nachfolgenden Termine klinische Kontrolluntersuchungen sowie Kaltluftprovokationen. Während der gesamten Studiendauer war Salbutamol-Dosieraerosol als Bedarfsmedikation erlaubt.

Dem Studienvorhaben wurde von der Ethik-Kommission der Medizinischen Fakultät Charité als ethisch vertretbar zugestimmt. Voraussetzung zur Teilnahme war die schriftliche Einverständniserklärung der Erziehungsberechtigten und der Studienteilnehmer.

\section{Tagebuch}

$\mathrm{Zu}$ den Vorstellungsterminen U1 und U3 wurden den Probanden Asthmatagebücher ausgehändigt, die über jeweils 10 Wochen zu führen waren. Notiert werden sollte der morgens und abends zu ermittelnde Peak-flow-Wert (Peak-flow-Messgerät CE, Range $500 \mathrm{l} / \mathrm{min}$, Fa. Hagedorn, Tellig) sowie der Verbrauch an Bedarfsmedikation als Anzahl der pro Tag inhalierten Hübe Sultanol ${ }^{\circledR}$ (Fa. Glaxo, Hamburg). Außerdem wurden die Kinder bzw. deren Eltern gebeten, für ihre Asthmabeschwerden (Husten, Giemen, Engegefühl im Brustkorb und Luftnot) während der Nacht und während des Tages jeweils 0 bis 3 Punkte zu vergeben. ( $0=$ keine, $1=$ leichte, $2=$ mittlere, 3 = starke Beschwerden).

\section{Kaltluftprovokation}

Die Kaltluftprovokation wurde wie folgt durchgeführt:

1. Ruhelungenfunktion im Bodyplethysmographen der Fa. Jäger, Würzburg

2. Isokapnische Hyperventilation (Beimischung von $5 \% \mathrm{CO}_{2}$ ) von $-12{ }^{\circ} \mathrm{C}$ kalter Luft über 4 Minuten (RHES, Fa. Jäger, Würzburg)

3. Nach 4 Minuten 2. Bodyplethysmographie

4. Bronchospasmolyse (2 Hübe Sultanol ${ }^{\circledR}$-Dosieraerosol über Inhalierhilfe)

5. Nach 10 Minuten 3. Bodyplethysmographie 


\section{Endpunkte}

Primäre Endpunkte der Untersuchung waren das $\mathrm{FEV}_{1} 4 \mathrm{~min}$ nach Inhalation von Kaltluft, der Verbrauch an Bedarfsmedikation (Anzahl der Hübe Salbutamol) sowie der morgendliche Peak-flow-Wert. Weitere Endpunkte waren Peak-flow und $\mathrm{MEF}_{25}$ in der Ruhelungenfunktion und nach Inhalation von Kaltluft, das Ansprechen auf die Inhalation mit Beta-Sympathomimetika und das Symptomenprotokoll.

\section{Statistik}

Für gepaarte Stichproben wurde der Wilcoxon-Test, für Gruppenvergleiche der Mann-Whitney-U-Test verwendet. Die statistische Analyse wurde unter Verwendung von SPSS/PC, Version 8.0.0, SPSS Inc., USA, durchgeführt.

\section{Ergebnisse}

\section{Patienten}

17 von 20 Kindern beendeten die Studie und wurden in die Auswertung eingeschlossen. Ausschluss-, bzw. Abbruchgründe waren Verweigerung der Einverständniserklärung durch den zweiten Elternteil, Exazerbation des Asthma bronchiale und mangelnde Compliance. Das Durchschnittsalter lag bei 8,5 Jahren, die durchschnittliche Erkrankungsdauer betrug 4,7 Jahre. 15 der 17 Kinder (13 Knaben, 4 Mädchen) litten unter einem allergischen Asthma bronchiale (positiver Hautpricktest, bzw. Nachweis von spezifischem IgE gegen mindestens ein Inhalationsallergen). Vor Beginn der klinischen Prüfung waren 2 Kinder mit DNCG, 3 mit Salbutamol, 10 mit einer Kombination aus DNCG und Beta-Sympathomimetikum und 2 mit einem topischen Steroid $(2 \times 50 \mu \mathrm{g}$ Fluticason) behandelt. 7 Patienten starteten mit Montelukast (Gruppe 1), 10 mit DNCG (Gruppe 2). Bei den Ausgangsbedingungen ließen sich keine signifikanten Unterschiede hinsichtlich demographischer Daten und Lungenfunktionswerten zwischen Gruppe 1 und Gruppe 2 ermitteln. Neben der Studienmedikation wurden außer Beta-Sympathomimetika als Bedarfsmedikation keine weiteren Medikamente verwendet. Über unerwünschte Begleitreaktionen bzw. Nebenwirkungen wurde in keinem Fall berichtet.

\section{Studiendesign}

Um nachzuweisen, ob die Ergebnisse gleicher Therapiephasen (nach Cross-over-bedingter Aufteilung) zusammengefasst werden können, wurden die Zielparameter miteinander verglichen. Dabei zeigten sich keine Unterschiede, so dass ein Effekt durch das Cross-over ausgeschlossen und die Ergebnisse im Weiteren zusammen $(n=17)$ betrachtet wurden.

\section{Kaltluftprovokation}

Um zu prüfen, ob die Methode der Kaltluftprovokation auch bei Kindern mit leichtem Asthma bronchiale genügend sensitiv ist, eine bronchiale Hyperreagibilität nachzuweisen, wurde der Einfluss dieser Provokationsmethode auf die Lungenfunktionswerte zum Zeitpunkt U2 und U4 unabhängig vom Therapieregime untersucht. Der Vergleich der Parameter $\mathrm{FEV}_{1}$ und $\mathrm{MEF}_{25}$ zwischen der Ruhelungenfunktion (A) und der zweiten Lungenfunktion nach Kaltluftprovokation (B) und der dritten Lungenfunktion nach Inhalation von Beta-Sympathomimetika (C) ergab bis auf eine Ausnahme $\left(\mathrm{MEF}_{25}\right.$ in $\mathrm{A}$ und B) durchweg signifikante Unterschiede $(\mathrm{p}<0,05)$.

\section{Lungenfunktion}

Die im Studienkollektiv ermittelten Lungenfunktionsdaten sind als Mittelwerte und Standardabweichung in Tab. 1 dargestellt. Nach 8-wöchiger Therapie mit Montelukast zeigte sich beim $\mathrm{MEF}_{25}$ in der Ruhelungenfunktion mit 67,8\% des altersentsprechenden Soll ein signifikant höherer Wert im Vergleich zu den Ausgangswerten ohne Therapie (59,1\%, $\mathrm{p}<0,05)$. Vergleicht man die Lungenfunktionswerte nach 8wöchiger regelmäßiger Inhalationstherapie mit DNCG mit den Ergebnissen nach 14-tägiger Runin-Phase, fallen signifikante Unterschiede bei den Parametern $\mathrm{FEV}_{1}$ und $\mathrm{MEF}_{25}$ sowohl in der Ruhelungenfunktion als auch nach Kaltluftprovokation auf.

Die Ergebnisse des $\mathrm{FEV}_{1}$ für die drei Zeitpunkte nach Runin, nach Montelukast und nach DNCG unter Einfluss der Kaltluftprovokation sind in Abb. 2a grafisch dargestellt. Für jedes Regime ergaben sich zwischen (A) und (B), zwischen (B) und (C) sowie zwischen (A) und (C) signifikante Unterschiede (jeweils $\mathrm{p}<0,05)$. Besonders deutlich wurden diese Unterschiede bei der Betrachtung von drei ausgewählten Studienpatienten, die in der U2 auf die Kaltluftprovokation mit einer Reduktion der $\mathrm{MEF}_{25}$ von mehr als 25\% (Abfall um 26\% bei Pat. 1, um 42,5\% bei Pat. 10 bzw. um 50,7\% bei Pat. 13) reagiert haben (Abb. 2b). Insgesamt ergaben sich zwischen beiden Prüfsubstanzen Montelukast und DNCG bei keinem der ermittelten Parameter signifikante Unterschiede.

Errechnet man die individuellen Differenzen (Delta) der Parameter $\mathrm{FEV}_{1}$ und $\mathrm{MEF}_{25}$ zwischen Ruhelungenfunktion (A) und nach Provokation (B), zwischen Ruhelungenfunktion (A) und nach Bronchospasmolyse (C), sowie zwischen den Daten, die nach Kaltluftprovokation (B) und nach Inhalation (C) und vergleicht diese zwischen den einzelnen Regimes (Runin, Montelukast, DNCG) ließen sich signifikante Unterschiede ausschließlich beim Delta zwischen $(A)$ und $(C)$ nachweisen. Gemäß Abb. 3 war das Delta des FEV $_{1}$ unter Montelukast mit

Tab. 1 Mittelwerte der Lungenfunktionsdaten (\% des altersentsprechenden Soll) im Vergleich

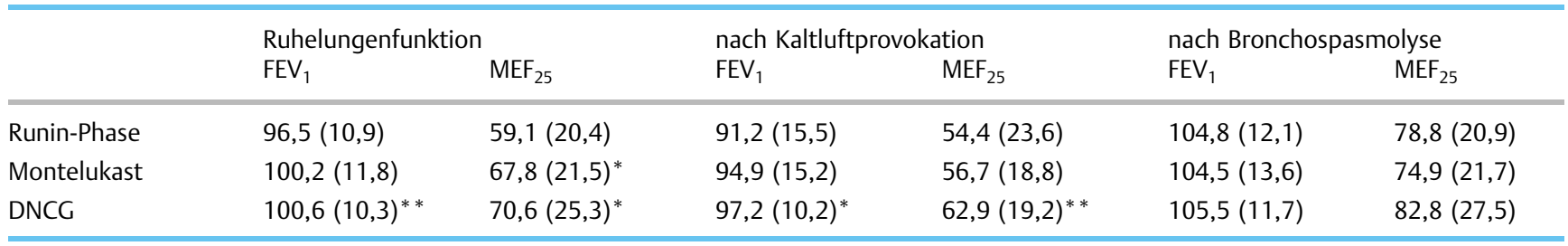

${ }^{*} \mathrm{p}<0,05 ;{ }^{* *} \mathrm{p}<0,01$, jeweils bezogen auf Runin-Phase. Standardabweichungen in Klammern. 


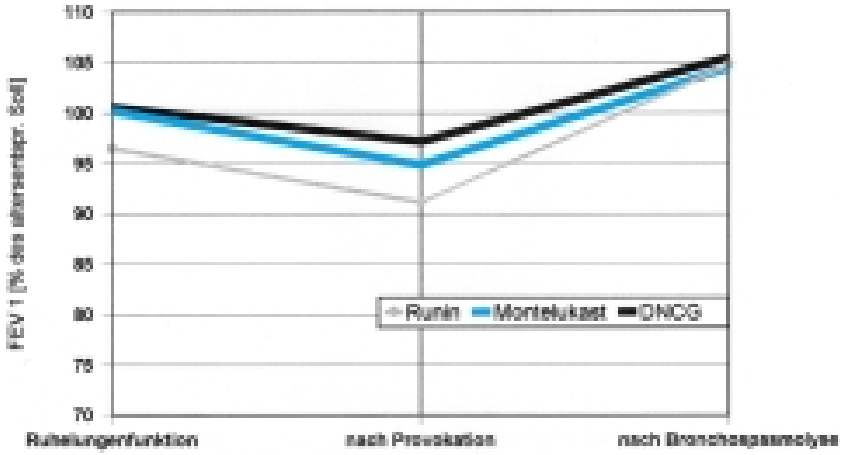

Abb. 2a $\mathrm{FEV}_{1}$ (Mittelwerte) unter Einfluss der Kaltluftprovokation.

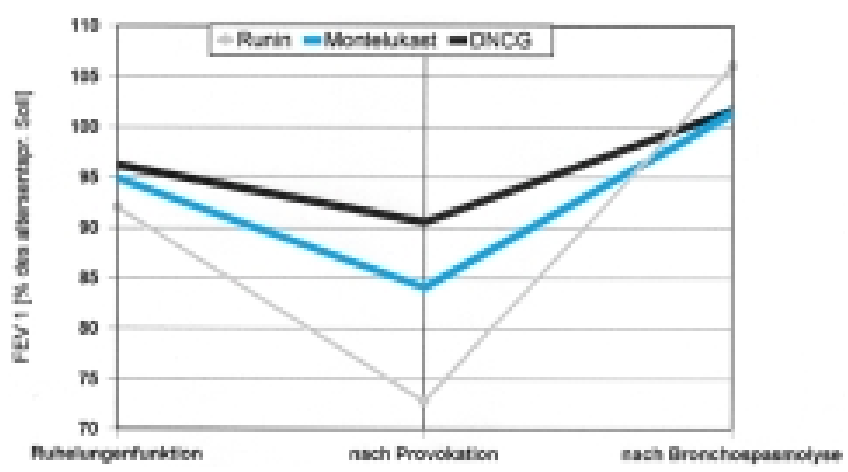

Abb. 2 b $\mathrm{FEV}_{1}$ (Mittelwerte) unter Einfluss der Kaltluftprovokation für 3 ausgewählte Studienpatienten, die in der 1. Kaltluftprovokation mit einer Reduktion des $\mathrm{MEF}_{25}$ von mehr als $25 \%$ reagiert haben.

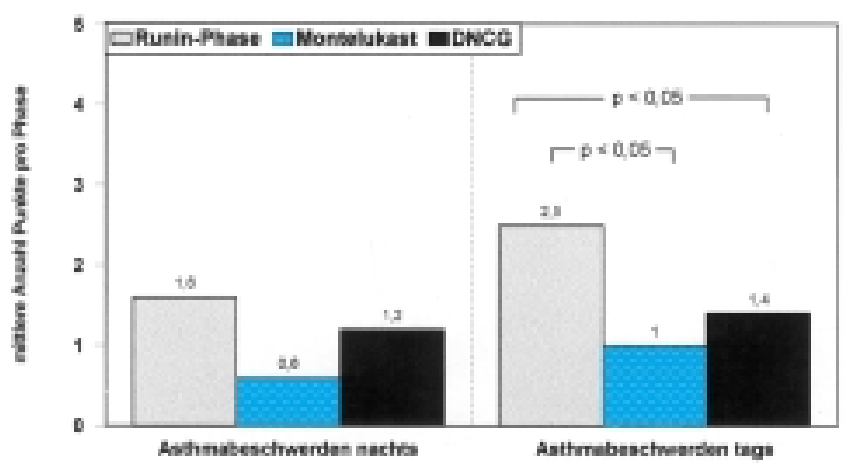

Abb. 3 Mittelwerte der individuellen Differenzen (Delta) von FEV und $\mathrm{MEF}_{25}$ zwischen Bronchospasmolyse (C) und Ruhelungenfunktion (A).

4,4 $\pm 7,1$ kleiner als ohne Medikation (8,2 $\pm 4,7$; $\mathrm{p}<0,05)$. Für den Parameter $\mathrm{MEF}_{25}$, war das Delta unter Montelukast mit $7,0 \pm 20,3$ ebenfalls kleiner als ohne Medikation (19,7 $\pm 15,2$; $\mathrm{p}<0,05)$. Das Delta des $\mathrm{FEV}_{1}$ unter DNCG war mit 4,8 $\pm 7,2$ nicht signifikant kleiner als in der Runin-Periode. Vergleichbar verhielt es sich mit dem $\operatorname{MEF}_{25}(12,2 \pm 22,2$ vs. 19,7).

\section{Asthmabeschwerden}

Aufgrund des überwiegend geringen Beschwerdeniveaus, wurden die von den Kindern für jeden Tag angegebenen Punkte über jeweils eine Woche summiert, so dass ein maximaler Score von 21 Punkten pro Woche sowohl für morgendliche als auch für abendliche Asthmabeschwerden erreicht werden konnte.

Gemäß Abb.4 ergab sich ein nächtlicher Wochenscore von durchschnittlich 1,6 $\pm 2,6$ in der Runin-Periode, von 1,2 $\pm 1,8$

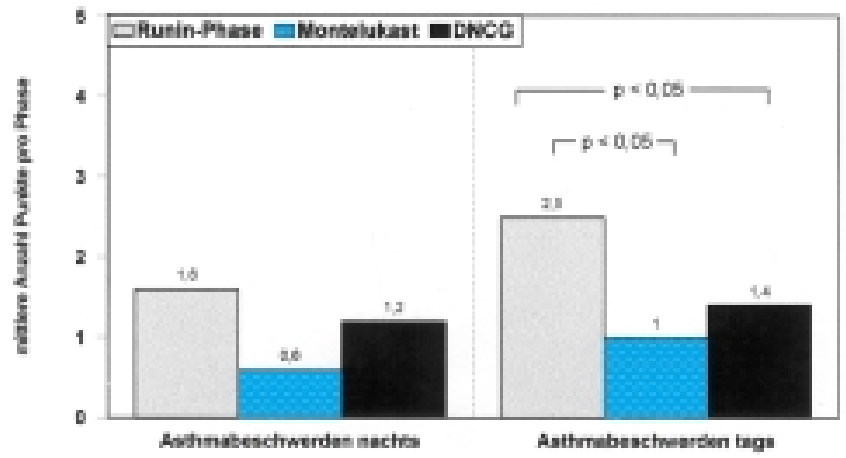

Abb. 4 Asthmabeschwerden während der Nacht und tagsüber als Punktescore. Pro Woche konnten max. 21 Punkte erreicht werden. Angegeben ist jeweils die mittlere Anzahl der pro Phase erreichten Punkte.

unter DNCG und von 0,6 \pm 1,0 unter Montelukast (n.s.). Das Beschwerdeniveau tagsüber wurde von den Patienten insgesamt etwas höher eingestuft als das während der Nacht. Signifikante Unterschiede ergaben sich sowohl zwischen Runin- (2,5 $\pm 2,7$ Punkte) und DNCG-Phase (1,4 \pm 1,9 Punkte), als auch zwischen Runin- und Montelukast-Phase $(1,0 \pm 1,6$ Punkte; $\mathrm{p}<0,05)$. Keine Unterschiede ließen sich zwischen Montelukast und DNCG ausmachen. Insgesamt war im Verlauf der Studie kein Trend erkennbar.

\section{Peak-flow}

Zur Auswertung der Peak-flow-Werte wurde die Peak-flowVariabilität für jeweils zwei Wochen einer jeden Phase (Runin-Therapie - Washout-Therapie) errechnet, in dem die Differenz aus dem höchsten und dem niedrigsten Wert zweier Wochen gebildet und schließlich durch den aus den 14 Eintragungen errechneten Mittelwert geteilt wurde. Diese Peak-flow-Variabilität wurde ebenfalls als Mittelwert für morgens und abends getrennt bestimmt (Tab. 2). Bei den 8wöchigen Therapiephasen wurden jeweils die letzten 14 Tage

Tab. 2 Mittelwerte der Peak-flow-Variabilität über je 2 Wochen

\begin{tabular}{lllll}
\hline & Runin & Montelukast & Washout & DNCG \\
\hline morgens & $29,6(15,6)$ & $23,7(17,8)$ & $21,5(13,3)$ & $23,8(11,9)$ \\
abends & $32,3(19,4)$ & $21,2(19,0)^{*}$ & $24,7(14,5)^{*}$ & $24,9(13,1)^{*}$ \\
\hline
\end{tabular}

* Wert signifikant $(\mathrm{p}<0,05)$ niedriger als in Runin-Periode (abends). Standardabweichungen in Klammern. 
zugrunde gelegt. Zwischen beiden Therapiephasen ergaben sich wiederum keine signifikanten Unterschiede; nur die Abendwerte - sowohl der Behandlungsphasen, als auch der Washout-Periode - lagen signifikant niedriger $(\mathrm{p}<0,05)$ als in der Runin-Phase.

\section{Bedarfsmedikation}

Der Verbrauch an Bedarfsmedikation wurde täglich als Anzahl der benötigten Hübe Sultanol ${ }^{\circledR}$ im Tagebuch dokumentiert. Durchschnittlich wurden in den vier Studienphasen 0,5-3,2 Hübe pro Woche benötigt. Dabei ergaben sich weder signifikante Unterschiede zwischen beiden Therapien noch im Vergleich zu den Perioden ohne Therapie.

\section{Diskussion}

Die Wirksamkeit von DNCG als prophylaktische Therapie bei leichtem Asthma bronchiale im Kindesalter ist hinlänglich evaluiert. Die vorliegende Pilotstudie wurde durchgeführt, um zu überprüfen, ob die Einnahme von Montelukast ebenfalls als Dauerprophylaxe eines kindlichen Asthma bronchiale geeignet ist. Nach Literaturrecherche existiert bislang keine Publikation, die DNCG mit Leukotrienrezeptorantagonisten hinsichtlich ihrer Wirksamkeit beim Einsatz als Monotherapie vergleicht. Lediglich ein Abstract [17] belegt die naheliegende Vermutung, dass die orale, einmal tägliche Einnahme einer Kautablette Singulair ${ }^{\circledR}$ einer viermal täglichen Inhalationstherapie mit DNCG-Dosieraerosol vorgezogen wird.

Vorliegende Untersuchung dokumentiert eine vergleichbar gute Kontrolle von Asthmasymptomen und Lungenfunktionswerten durch DNCG und Montelukast. Weder bei den objektiven Parametern $\left(\mathrm{FEV}_{1}\right.$ und $\mathrm{MEF}_{25}$ in Ruhe, nach Kaltluftprovokation und nach Bronchospasmolyse) und dem Peak-flowProtokoll noch bei den subjektiven Parametern (Beschwerdescore, Verwendung von Bedarfsmedikation) ließen sich relevante Unterschiede zwischen beiden Medikamenten ermitteln. Trotz fehlender Unterschiede im direkten Vergleich beider Präparate führte Montelukast im Vergleich zur RuninPeriode ohne Medikation lediglich bei einem Parameter zu einem signifikanten Unterschied $\left(\mathrm{MEF}_{25}\right.$ in der Ruhelungenfunktion), während DNCG sowohl in der Ruhelungenfunktion als auch nach Kaltluftprovokation bei beiden Zielparametern $\left(\mathrm{FEV}_{1}\right.$ und $\left.\mathrm{MEF}_{25}\right) \mathrm{zu}$ besseren Ergebnissen führte. Hierbei ist jedoch die vergleichsweise niedrige Fallzahl zu berücksichtigen.

Zur Wirksamkeit von DNCG finden sich in der Literatur unterschiedliche Ergebnisse. So erwies sich die regelmäßige Inhalationstherapie von dreimal $10 \mathrm{mg}$ DNCG-Dosieraerosol über Spacer bei ein- bis vierjährigen Kindern in Bezug auf die Anzahl symptomfreier Tage nach 5-monatiger Therapiedauer als nicht effektiver als Plazebo [18]. Der Einfluss von DNCG auf die bronchiale Hyperreagibilität ist in etlichen Studien mit unterschiedlichen Ergebnissen dokumentiert worden. Während die Inhalation von viermal $20 \mathrm{mg}$ DNCG über 16 Wochen bei 44 Asthmatikern nicht zu einer Verbesserung von Peak-flow-Werten, $\mathrm{PC}_{20} \mathrm{FEV}_{1}$ für Histamin und Bedarf an Rescuemedikation führte [19], war diese Therapie in einer weiteren Arbeit [20] in der Lage, die bronchiale Hyperreagibilität, gemessen an der $\mathrm{PD}_{35} \mathrm{SGAW}$ nach Histaminprovokation signifikant zu senken. Außerdem konnte im Vergleich zu
Plazebo ein signifikanter Schutz vor pollen-assoziierter bronchialer Hyperreagibilität bei 22 Patienten demonstriert werden [21]. Darüber hinaus erwies sich DNCG (10 mg/d) nach 12 Wochen Therapie als ebenso wirksam wie Nedocromil-Natrium ( $4 \mathrm{mg} / \mathrm{d})$ und Beclometason-Dipropionat $(500 \mu \mathrm{g} / \mathrm{d})$, gemessen an der $\mathrm{PD}_{20} \mathrm{FEV}_{1}$ nach Metacholin-Provokation [22]. Andere Studien kommen beim Vergleich zwischen inhalativen Steroiden und DNCG jedoch $\mathrm{zu}$ weniger eindrucksvollen Ergebnissen. Bei kindlichem Asthma bronchiale führte Beclometason-Dipropionat zu einer Verbesserung der unspezifischen bronchialen Hyperreagibilität nach Carbachol um den Faktor 5,9, DNCG lediglich um den Faktor 1,9 [23]. Einem Review [24] zu Folge führt die regelmäßige Inhalationstherapie mit DNCG insgesamt jedoch zu einer Verringerung bronchialer Hyperreagibilität bei atopischen und nicht-atopischen Asthmatikern aller Altersgruppen. Unter Berücksichtigung der kleinen Fallzahl kommt auch die vorliegende Untersuchung zu diesem Ergebnis.

Bereits die ersten Erwachsenenstudien belegten, dass Leukotrienrezeptorantagonisten in der Lage sind, vor belastungsabhängiger Bronchokonstriktion zu schützen [25]. Später wurde von Leff [9] gezeigt, dass es nach 12-wöchiger Einnahme von Montelukast bei 97 Erwachsenen zu einem deutlich geringeren belastungsinduzierten $\mathrm{FEV}_{1}$-Abfall kam. Für das Kindesalter existieren bislang Daten in nur begrenztem Umfang. Bei 6- bis 14-jährigen Kindern konnte nach 2-tägiger Einnahme von Singulair $^{\circledR}$ ein Schutz vor belastungsinduzierter Bronchokonstriktion gezeigt werden [16]. Auch bei jüngeren Kindern (3-5 Jahre) führte die 2-tägige Einnahme von je $5 \mathrm{mg}$ Montelukast zu einer deutlich geringeren kaltluftinduzierten Bronchokonstriktion gemessen an spezifischen Atemwegswiderstand im Vergleich zu Plazebo (17 versus 46\%), unabhängig davon, ob begleitend inhalative Steroide eingenommen wurden oder nicht [26]. Zu einer Verbesserung des morgendlichen $\mathrm{FEV}_{1}$ um 8,2\% gegenüber den Ausgangswerten (Plazebo $3,6 \%$ ) kam es nach 8-wöchiger Einnahme von Montelukast bei 336 Kindern gleicher Altersgruppe [15]. Auch die Einnahme eines weiteren Leukotrienrezeptorantagonisten (Zafirlukast) führte bei 6- bis 14-jährigen Asthmatikern zu einer geringeren Reagibilität der Atemwege nach Laufbandbelastung [27].

Diese Pilotstudie gibt Anhalt dafür, dass die 8-wöchige Einnahme von Montelukast zu einer signifikanten Verbesserung der $\mathrm{MEF}_{25}$ in der Ruhelungenfunktion im Vergleich $\mathrm{zu}$ den Ausgangsdaten führen kann. Die Variabilität der Peak-flowWerte fiel im Vergleich zur Runin-Phase geringer aus und erreichte bei den Abendmessungen statistische Signifikanz. Ein weiterer Endpunkt dieser Untersuchung waren die Asthmabeschwerden, die unter Therapie mit 0,6 bis 1,4 von 21 maximal pro Woche erreichbaren Punkten subjektiv als sehr gering eingestuft wurden. Unter Berücksichtigung der Einschlusskriterien war dies zwar kaum anders zu erwarten, dennoch lag das Beschwerdeniveau (tags) signifikant unter dem ohne Therapie, unabhängig davon, welche der beiden Prüfsubstanzen eingenommen wurde. In anderen Studien [11,15], in welchen ebenfalls ein Symptomen-Score als Endpunkt gewählt wurde, ließen sich hingegen keine Unterschiede zwischen Montelukast und Plazebo nachweisen.

Die dem kindlichen Asthma bronchiale zugrunde liegende bronchiale Hyperreagibilität kann mit Hilfe der Kaltluftpro- 
vokation nachgewiesen werden [28,29]. Es gibt Hinweise, dass diese Art bronchialer Provokation die Pathophysiologie des Asthma bronchiale besser widerspiegelt, als die durch Histamin oder Metacholin medikamentös ausgelöste Bronchokonstriktion [30]. Nielsen und Bisgaard haben kürzlich über eine Spezifität der Kaltluftprovokation von $93 \%$ bei $2-$ bis 5-jährigen Asthmatikern berichtet [31]. Bei unserem Studienkollektiv führte die Kaltluftprovokation zwar rechnerisch überwiegend zu signifikanten Unterschieden, dennoch bleibt offen, inwieweit bei einem mittleren $\mathrm{FEV}_{1}$-Abfall um 5,3\% und des $\mathrm{MEF}_{25}$ um 4,7\% überhaupt von einer relevanten bronchialen Hyperreagibilität ausgegangen werden kann. Vergleicht man die $\mathrm{FEV}_{1}$-Werte aller Probanden mit einer Subgruppe von drei ausgewählten Studienpatienten, die am ausgeprägtesten auf die Kaltluftprovokation reagiert haben, zeigt sich, dass das Gesamtkollektiv einen offensichtlich zu geringen Schweregrad aufwies. Um die Wirksamkeit von Leukotrienrezeptorantagonisten als Monotherapie bei mildem Asthma bronchiale genauer zu evaluieren, erscheint es demnach sinnvoller, sich bei der Auswahl der Einschlusskriterien nicht nur auf anamnestische Angaben zu Beschwerdeniveau sowie Art und Umfang prophylaktischer Medikation zu beziehen, vielmehr müsste ergänzend ein Schweregrad anhand der Kaltluftprovokation zugrunde gelegt werden.

Ohne Gegenstand vorliegender Untersuchung zu sein, liefert eine Reihe von Studien Evidenz zu Arzneimittelsicherheit und Wirksamkeit von Leukotrienrezeptorantagonisten. Im Gegensatz zu DNCG ist deren Rolle in der Behandlung des kindlichen Asthma bronchiale bis jetzt nicht abschließend definiert. Vorliegende Pilotstudie zeigt, dass Montelukast auch bei leichtem Asthma bronchiale als Dauerprophylaxe geeignet sein kann. Studien mit größeren Fallzahlen sind jedoch erforderlich, die bisherige Empfehlung als „add-on“-Präparat zu ergänzen, um Montelukast als Monotherapie bei leichtem Asthma bronchiale einzusetzen.

\section{Literatur}

${ }^{1}$ National Heart, Lung and Blood Institute. National Asthma Education an Prevention Program. Expert Panel Report 2: guidelines for the diagnosis and management of asthma. In: NIH Publication No. 97 - 4051. Bethesda, MD: National Institute of Health, 1995: 1-48

2 Shapiro GG, Sharpe M, DeRouen TA, Pierson WE, Furukawa CT, Virant FS, Bierman CW. Cromolyn versus triamcinolone acetonide for youngsters with moderate asthma. J Allergy Clin Immunol 1991; 88: $742-748$

${ }^{3}$ Blumenthal MN, Selcow J, Spector S, Zeiger RS, Mellon M. A multicenter evaluation of the clinical benefits of cromolyn sodium aerosol by metered-dose inhaler in the treatment of asthma. J Allergy Clin Immunol 1988; 81: 681-687

${ }^{4}$ Kelloway JS, Wyatt RA, Adlis SA. Comparison of patients' compliance with presribed oral and inhaled asthma medications. Arch Int Med 1994; 154 (12): 1349-1352

${ }^{5}$ FDA. Joint Meeting of the Pulmonary and Allergy Drug Advisory and the Endocrine and Metabolic Drig Advisory Committees. [Transcript] Jul 28 - 29; Day\#2:16, 1998

${ }^{6}$ Busse WW. The role of leukotrienes in asthma and allergic rhinitis. Clin Exp Allergy 1996; 26: 868 -879

${ }^{7}$ Diamant Z, Hiltermann JT, Van Rensen EL. The effect of inhaled leukotriene D4 and metacholine on sputum cell differentials in asthma. Am J Respir Crit Care Med 1997; 155: 1247-1253
${ }^{8}$ Wenzel SE. Arachidonic acid metabolites: mediators of inflammation in asthma. Pharmacotherapy 1997; 17: 3S-12S

${ }^{9}$ Leff JA, Busse WW, Pearlman D. Montelukast, a leukotriene receptor antagonist, for the treatment of mild asthma and exercise-induced bronchoconstriction. N Engl J Med 1998; 339: $147-152$

${ }^{10}$ Noonan MJ, Chervinsky P, Brandon M. Montelukast, a potent leukotriene receptor antagonist, causes dose-related improvement in chronic asthma. Eur Respir J 1998; 11: 1232 - 1239

${ }^{11}$ Reiss TF, Chervinsky P, Dockhorn RJ, Shingo S, Seidenberg B, Edwards TB. Montelukast, a once-daily leukotriene receptor antagonist in the treatment of chronic asthma. Arch Intern Med 1998; 158: $1213-1219$

12 Spector SL, Smith LJ, Glass M and the accolate Asthma Trialist Group. Effect of 6 weeks of therapy with oral doses of ICI 204, 219, a leukotriene D4 receptor antagonist, in subjects with bronchial asthma. Am J Respir Crit Care Med 1994; 150: 618623

${ }^{13}$ Diamant Z, Timmers MC, v/d Veen H, De Smet M, Leff JA, Friedmann BS. Effect of oral Montelukast (MK-0476), a potent leukotriene receptor antagonist, on allergen-induced airway responses in asthmatic subjects. Am J Respir Crit Care Med 1996; 153: A346

${ }^{14}$ Reiss TF, Hill JB, Harman E, Zhang J, Tanake WK, Bronsky E. Increased urinary excretion of LTE4 after exercise and attenuation of exercise-induced bronchospasm by montelukast, a cysteinyl leukotriene receptor antagonist. Thorax 1997; 52: 1030 1035

${ }^{15}$ Knorr B, Matz J, Bernstein JA, Nguyen H, Seidenberg BC, Reiss TF, Becker A for the Pediatric Montelukast Study Group. Montelukast for chronic asthma in 6- to 14-year-old children. JAMA 1998; 279: 1181 - 1186

${ }^{16}$ Kemp JP, Dockhorn RJ, Shapiro G, Nguyen H, Reiss TF, Seidenberg BC, Knorr B. Montelukast once daily inhibits exercise-induced bronchoconstriction in 6- to 14-year-old children with asthma. J Pediatr 1998; 133: 424-228

${ }^{17}$ Edelman JM, Milewski KA, Turpin JA, Santanello NC, Bird SR, Radler CA, Horsham PA. Effectiveness and safety of montelukast, a leukotriene receptor antagonist, compared to inhaled cromolyn in moderate asthmatic children ages 6 to 11. J Allergy Clin Immunol 1999; 103: S134

${ }^{18}$ Tasche MJA, v/d Wouden JC, Uijen JHJM, Ponsioen BP, Bernsen RMD, v Suijlekom-Smit LWA. Randomised placebo-controlled trial of inhaled sodium cromoglycate in 1-4-year-old children with moderate asthma. Lancet 1997; 350: 1060-1064

${ }^{19}$ Jenkins CJ, Breslin AB. Long term study of the effect of sodium cromoclycate on non-specific bronchial hyperresponsivness. Thorax 1987; 42 (9): 664-669

${ }^{20}$ Chhabra SK, Gaur SN. Effect of long-term treatment with sodium cromoglycate on nonspecific bronchial hyperresponsiveness in asthma. Chest 1989; 95 (6): 1235-1238

${ }^{21}$ Lowhagen O, Rak S. Modification of bronchial hyperreactivity after treatment with sodium cromoglycate during pollen season. J Allergy Clin Immunol, 1985

22 Orefice U, Struzzo P, Dorigo R, Peratoner A. Long-term treatment with sodium cromoglycate, nedocromil sodium and beclomethasone dipropionate reduces bronchial hyperresponsiveness in asthmatic subjects. Respiration 1992; 59 (2): 97 - 101

${ }^{23}$ Kraemer R, Sennhauser F, Reinhardt M. Effects of regular inhalation of beclomethasone dipropionate and sodium cromoglycate on bronchial hyperreactivity in asthmatic children. Acta Paediatr Scand 1987; 76 (1): 119-123

${ }^{24}$ Hoag JE, McFadden ER. Long-term effect of cromolyn sodium on nonspecific bronchial hyperresponsiveness: a review. Ann Allergy $1991 ; 66(1)$ : $53-63$ 
${ }^{25}$ Manning PJ, Watson RM, Margolskee DJ. Inhibition of exerciseinduced bronchoconstriction by MK-571, a potent leukotriene D4-receptor antagonist. N Engl J Med 1990; 232: 1736-1739

${ }^{26}$ Bisgaard H, Nielsen KG. Bronchoprotection with a leukotriene receptor antagonist in asthmatic preschool children. Am J Respir Crit Care Med 2000; 162: 187-190

${ }^{27}$ Pearlman DS, Ostrom NK, Bronsky EA, Bonuccelli CM, Hanby LA. The leukotriene D4-receptor antagonist zafirlukast attenuates exercise induced bronchoconstriction in children. J Pediatr 1999; 134: $273-279$

${ }^{28}$ Wjst M, Dold S, Reitmeir P, Wulff A, Nicolai T, v. Mutius E. Evaluation of cold air challenge data in a population sample using a model of bronchial hyperreactivity and disposition to bronchial obstruction. Pediatr Pulmonol 1993; 15: 339- 344

${ }^{29}$ Reisman J, de Benedictis F, McLaughlin J, Levison H. Cold air challenge in children with asthma. Pediatr Pulmonol 1987; 3: $251-254$

${ }^{30}$ Zach MS. Cold dry air challenge for measuring bronchial hyperresponsiveness - where do we stand? Pediatr Pulmonol 1995; 19: $323-325$

${ }^{31}$ Nielsen KG, Bisgaard $\mathrm{H}$. Lung function response to cold air challenge in asthmatic and healthy children of 2-5 years. Am J Respir Crit Care Med 2000; 161: 1805-1809

\section{PD Dr. B. Niggemann}

Charité - Campus Virchow-Klinikum

Abt. für Pädiatrische Pneumologie und Immunologie

Augustenburger Platz 1

13353 Berlin

E-mail: bodo.niggemann@charite.de

\section{INFORMATION}

Der jährliche Informationsbericht des DZK ist soeben erschienen. Er enthält die Daten zur Tuberkulose-Epidemiologie in Deutschland bis 1999 und kann ab sofort zum Preis von DM 32,- zzgl. 7\% Mwst. und Porto bezogen werden.

Prof. Dr. R. Loddenkemper

Generalsekretär

Lungenklinik Heckeshorn

Zum Heckeshorn 33

14109 Berlin 